\title{
Establishing the HLS-M-Q18 short version of the European Health Literacy survey questionnaire for the Malaysian context
}

\begin{abstract}
Background: The European Health Literacy Survey Questionnaire (HLS-EU-Q47) is becoming a widely used tool to measure health literacy (HL), including in Malaysia. There are efforts to reduce the 47-item scale to parsimonious short item scales that still reflect the assumptions and requirements of the conceptual model. This study used confirmatory factor analysis to reduce the 47-item scale to a short scale that can offer a feasible HL screening tool with sufficient psychometric properties. Methods: A cross-sectional survey was conducted on the Malaysian population based on ethnic distribution to ensure that the short version instrument reflects the country's varied ethnicities. The survey was administered by well-trained interviewers working for the Ministry of Health Malaysia. A total of 866 responses were obtained. Data was analysed using multi-factorial confirmatory factor analysis (CFA) with categorical variables. Results: The analysis resulted in a satisfactory 18 -item model. There were high correlations among the 18 items. The internal consistency reliability was robust, with no floor/ceiling effects. These results represented equivalence and consistency among the responses to items, suggesting that these items were homogenous in measuring Malaysian health literacy. The strong convergent and discriminant validity of the model makes the proposed 18 items a suitable short version of the health literacy instrument for Malaysia. Conclusions: The researchers propose the 18 -item instrument to be named HLS-M-Q18. This short version instrument may be used in measuring health literacy in Malaysia as it achieved robust reliability, structural validity and construct validity that fulfilled goodness-of-fit criteria.
\end{abstract}

Keyword: Health literacy; HLS-M-Q18; Short version instrument; HLS-EU-Q4; Instrument item reduction 